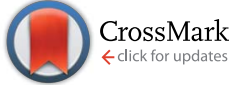

Cite this: Chem. Sci., 2016, 7, 945

Received 12th August 2015

Accepted 23rd October 2015

DOI: $10.1039 / c 5 s c 02992 a$

www.rsc.org/chemicalscience

\title{
s-Heptazine oligomers: promising structural models for graphitic carbon nitride $\uparrow$
}

\author{
A. Zambon, ${ }^{a b c}$ J.-M. Mouesca, ${ }^{\text {ab }}$ C. Gheorghiu, ${ }^{a b}$ P. A. Bayle, ${ }^{\text {ab }}$ J. Pécaut, ${ }^{\text {ab }}$ M. Claeys- \\ Bruno, $^{\text {d S. Gambarelli*ab and L. Dubois }}{ }^{* a b}$
}

Graphitic carbon nitride ( $\mathrm{g}-\mathrm{CN}$ ) has interesting catalytic properties but is difficult to study due to its structure and how it is produced. In this study, linear s-heptazine oligomers were synthesized to serve as well-defined molecular models for g-CN. Cyclic voltammetry, absorption and emission spectroscopies showed a clear shift of properties towards those of $\mathrm{g}-\mathrm{CN}$ as the number of heptazine units increased. DFT calculations supported the characterizations, and helped refine the properties observed.

\section{Introduction}

Carbon nitride (CN) was first synthesized by Berzelius and Liebig $^{1}$ in the early 19th century, making it one of the oldest polymers to have been studied. Its graphitic phase, g-CN, is an organic semi-conductor with a gap of around $2.7 \mathrm{eV}$ which has recently attracted considerable attention due to its photocatalytic properties. In addition to being capable of water splitting and a wide range of other important catalytic reactions, ${ }^{2}$ g-CN also has unique properties in numerous other domains, both in its bulk state and as nanoscale particles. ${ }^{3}$ Although first synthesized many decades ago, g-CN is still not well characterized. Its general structure was shown to be based on a tri-s-triazine or heptazine core,${ }^{4}$ but its three-dimensional structure remains elusive. Initially, g-CN was considered to be a layered 2D material (hence its name), but more recent studies suggest that it is more likely to be formed of linear polymeric chains, named melon or polymeric carbon nitride (PCN) (Chart 1a). ${ }^{5}$ These chains would then assemble to create layers. ${ }^{5}$ Study and use of g-CN is hampered by its poor solubility - which prevents the use of classical characterization techniques - and by the fact that alterations to experimental conditions during its synthesis (precursors, temperature, etc.) yield different materials. To help understand the structure-property relationships of this outstanding material it would be very useful to be able to synthesize well-defined, tailored molecular models for g-CN.

${ }^{a}$ Univ. Grenoble Alpes, INAC-SCIB, F-38000 Grenoble, France. E-mail: serge. gambarelli@cea.fr; lionel.dubois@cea.fr

${ }^{b} C E A$, INAC-SCIB, F-38000 Grenoble, France

${ }^{c}$ Aix Marseille Université, LISA EA4672, 13397 Cedex 20, Marseille, France

${ }^{d}$ French Environment and Energy Management Agency 20, avenue du Grésillé-BP 90406 49004, Angers Cedex 01, France

$\dagger$ Electronic supplementary information (ESI) available: Experimental procedures, DFT calculations and physical data. CCDC 1051347 and 1051348. For ESI and crystallographic data in CIF or other electronic format see DOI: $10.1039 / \mathrm{c} 5 \operatorname{sc} 02992 \mathrm{a}$
This type of strategy has previously been successfully used to elucidate the characteristics and fine-tune other materials such as graphene. ${ }^{6}$ If a method to synthesize low molecular weight analogues of g-CN were found it would provide access to a g-CN (heptazine oligomers) on a range of scales, from the bulk material down to individual molecules. Recently, the photocatalytic properties of melem (heptazine monomer) and a partially characterized low-mass oligomeric heptazine obtained from PCN by solvent extraction were published. ${ }^{7}$ This article has re-kindled interest in this class of molecules, and has made the need to obtain well-defined heptazine oligomers more pressing. In addition, to contribute to g-CN characterization, the chemistry based on these molecules could be useful in developing new families of ligands with novel physico-chemical properties (fluorescence, absorption, redox, etc.).

Because the chemistry of heptazine has not yet attracted much attention, heptazine oligomer synthesis is challenging. ${ }^{8} \mathrm{~A}$ key problem is that the most widely available and best described precursor material (cyameluric chloride) has three equivalent, very good, leaving groups which must be selectively exchanged by nucleophilic substitution during synthesis. Thus, selective substitution of only one or two chlorine atom(s) is difficult, ${ }^{9}$ and although reactions with secondary or primary amines have been reported in the literature, they generally lack selectivity. To overcome this hurdle, we chose diisobutylamine as the

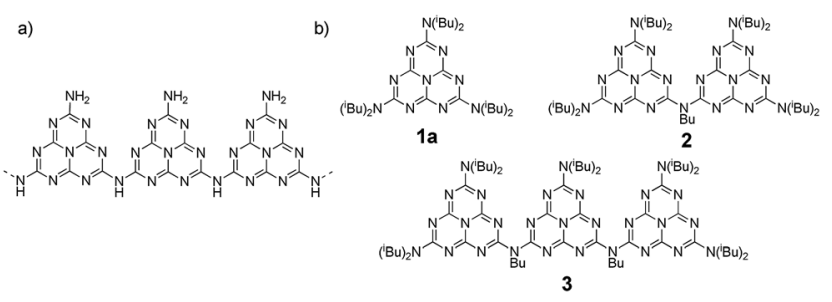

Chart 1 (a) proposed structure of PCN and (b) linear oligomers synthesized for this study. 


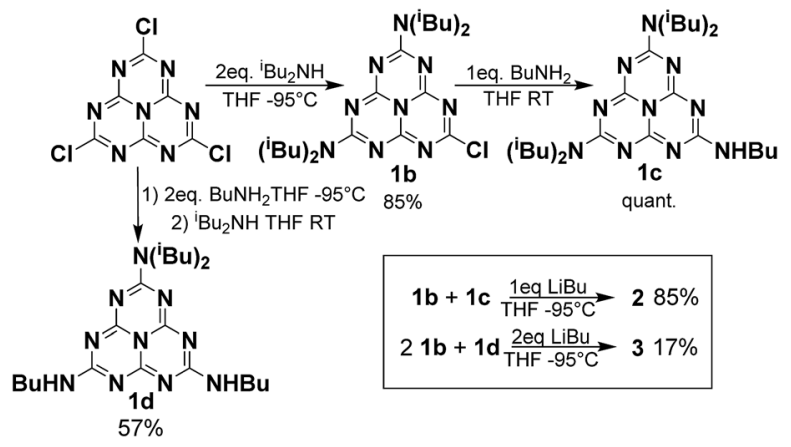

Scheme 1 Synthesis of heptazine oligomers.

functional group for peripheral positions; in addition to enhancing selectivity, diisobutyl substituents also result in a final molecule which is more soluble in organic solvents.

In this paper, we present the first report of synthesis of a soluble linear heptazine monomer 1a, dimer 2 and trimer 3 (Scheme 1 and Chart 1b); we also describe their extensive physicochemical characterization and associated DFT analysis.

\section{Results and discussion}

\section{Synthesis and characterisation}

Monomer 1a was synthesized from cyameluric chloride simply by adding a large excess of diisobutylamine at room temperature. Dimer $\mathbf{2}$ and trimer $\mathbf{3}$ were synthesized in two steps. Firstly, the primary and secondary amines were selectively substituted at low temperature to obtain dissymmetric heptazines $\mathbf{1 b}, \mathbf{1 c}$ and $\mathbf{1 d}$ (Scheme 1). Compounds $\mathbf{1 c}$ and $\mathbf{1 b}$ were then deprotonated with butyl lithium to generate a lithium amide which can substitute for the remaining chlorine atom on $\mathbf{1 b}$. To produce $\mathbf{3}$, two successive coupling reactions were necessary, which is possibly why the yield for this compound $(17 \%)$ was lower than the yield for $2(85 \%)$. All these compounds, unlike melem and cyameluric chloride, were relatively soluble in a large variety of organic solvents (including acetonitrile, tetrahydrofuran, chloroform, and hexane). This feature facilitates their characterization (see ESI $\dagger$ ). The expected structures of both the heptazine core and the alkyl moieties were fully compatible with IR, ${ }^{1} \mathrm{H}$ and ${ }^{13} \mathrm{C}$ liquid NMR spectra (Fig. S1-S20†). ${ }^{8 a, 9,10}$ XPS spectra of the as synthesized 1a, 2 and $\mathbf{3}$ oligomers were recorded (Fig. S58-S60†) and are in good agreement with data reported in literature for $\mathrm{g}-\mathrm{CN}$. $^{7}$

X-ray diffraction was performed on single crystals obtained by slow evaporation of a hexane/dichloromethane solution for 1a, and of a nitrobenzene solution for 2 (CCDC numbers 1051347 and 1051348, respectively) (see Table S1 for details†). The structure of 1a is shown in Fig. S26. $\dagger$ As previously reported for melem and its derivatives, the atoms of the heptazine system were found to be coplanar., ${ }^{9,10}$ The three bonds around the peripheral nitrogen (bearing the di-isobutyl substituent) were coplanar with the heptazine core, indicating an $\mathrm{sp}_{2}$ hybridization and a strong delocalization of the lone nitrogen electron pair onto the heptazine (mean deviation around the mean plane of the heptazine and nearby nitrogen: $0.043 \AA$ ). An $n-\pi$ interaction, with a distance of $3.45 \AA$, was observed between a peripheral nitrogen and the heptazine core of a nearby molecule. This distance is slightly larger than the interlayer distance measured by powder XRD for g-CN $(3.2 \AA) .{ }^{5}$

The mercury drawing for 2 indicates that the three bonds around the bridging nitrogen are coplanar (plane Nb) (Fig. 1). One of the key features of this structure is the wide angle $\left(66.7^{\circ}\right)$ between the two heptazine planes ( $\mathrm{H} 1$ and $\mathrm{H} 2)$. Plane $\mathrm{Nb}$ is located between $\mathrm{H} 1$ and $\mathrm{H} 2$, and the respective angles are NbH1: $50.26^{\circ}$ and $\mathrm{NbH2}: 22.67^{\circ}$. (Molecular) DFT calculations were performed on dimer 2 in an isolated state (i.e., without crystal packing). This analysis found the angle between the two heptazine units (calculated value $41.7^{\circ}$ ) to be the result of antagonistic behaviors: mutual repulsion between the lone-pair electrons of the core nitrogen atoms located close to the bridging bond and the $\pi$-delocalization through the bridging nitrogen. Further analysis indicated a low rotational barrier around this dihedral angle $\left(12.5 \mathrm{~kJ} \mathrm{~mol}^{-1}\right)$ (Table S11†). In stark contrast, the experimental structure for $\mathbf{1 a}$ and $\mathbf{2}$ showed lone pairs for all peripheral nitrogen atoms perpendicular to their respective heptazine planes. This result once again agreed closely with DFT which calculated a high rotational barrier (107 $\mathrm{kJ} \mathrm{mol}^{-1}$, Table S6†). To determine experimental values for these rotational barriers, ${ }^{1} \mathrm{H}$ and ${ }^{13} \mathrm{C}$ NMR experiments were performed for oligomers 2 and 3 in toluene at temperatures ranging from $223 \mathrm{~K}$ to $353 \mathrm{~K}$ (Fig. S23-S25 $\dagger$ ). For the isobutyl groups, only two distinct sets of ${ }^{1} \mathrm{H}$ signals were detected at low temperatures. These signals became equivalent for 2 at high temperatures, but the coalescence temperature was not reached for 3 in our experiments. Eyring analysis ${ }^{11}$ of 2 indicated an estimated rotational barrier of $78 \mathrm{~kJ} \mathrm{~mol}^{-1}$ (see ESI for calculation details $\dagger$ ), which is consistent with values published for ethyl groups in a dissymmetric monomeric melem derivative $\left(90 \mathrm{~kJ} \mathrm{~mol}^{-1}\right)^{9}$ and with our DFT calculations for $\mathbf{1 a}$ and dimer 2 (see above and ESI $\dagger$ ). These results suggest that the rotational barriers determined based on NMR data were those of the peripheral nitrogen groups. Almost free rotation around the bridging nitrogen produces symmetrical NMR spectra, leaving only two sets of distinct isobutyl groups.

\section{Optical properties}

The optical properties of $\mathbf{1 a}, \mathbf{2}$ and $\mathbf{3}$ in acetonitrile were studied by absorption and emission spectroscopies at room temperature (Fig. 2 and S27-S32†). The UV absorption spectra show
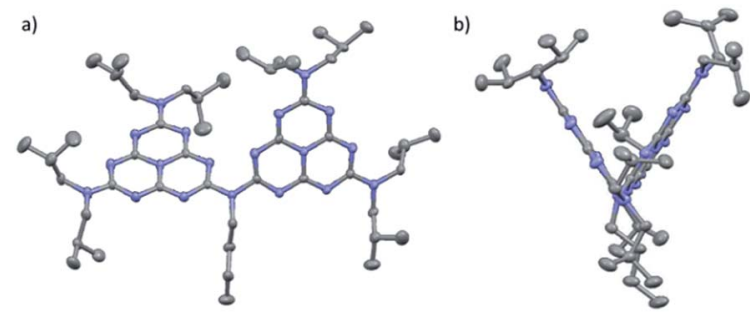

Fig. 1 (a) Front and (b) side mercury representations of 2 with $50 \%$ probability ellipsoids, based on single-crystal X-ray analysis (nitrobenzene molecules and hydrogen removed). 
a characteristic shape: a high absorbance zone and a mediumrange absorbance tail in the near-UV range. Molar extinction coefficients were $89400 \mathrm{M}^{-1} \mathrm{~cm}^{-1}$ for $1 \mathrm{a}(273 \mathrm{~nm}), 90200 \mathrm{M}^{-1}$ $\mathrm{cm}^{-1}$ for $2(276 \mathrm{~nm})$ and $111300 \mathrm{M}^{-1} \mathrm{~cm}^{-1}$ for $3(272 \mathrm{~nm})$. The lowest energy transitions were found at $315 \mathrm{~nm}(\varepsilon=$ $\left.2000 \mathrm{M}^{-1} \mathrm{~cm}^{-1}\right)$ for $1 \mathrm{a}, 368 \mathrm{~nm}\left(2400 \mathrm{M}^{-1} \mathrm{~cm}^{-1}\right)$ for 2 and $370 \mathrm{~nm}\left(4900 \mathrm{M}^{-1} \mathrm{~cm}^{-1}\right)$ for 3, indicating a significant bathochromic shift between 1a and $2(+53 \mathrm{~nm})$, and a very small one between 2 and $3(+2 \mathrm{~nm})$. A similar bathochromic shift between $1 \mathrm{a}$ and $2(+48 \mathrm{~nm})$ was found by DFT, but between compounds 2 and 3 , the shift calculated $(+30 \mathrm{~nm})$ was larger.

Like UV/Vis spectra, emission spectra (Fig. S43†) showed a bathochromic shift of the peak maximum between 1a (382 $\mathrm{nm}), 2$ (417 $\mathrm{nm})$ and $3(443 \mathrm{~nm})$. Emission spectra were not altered by changing the excitation wavelength, suggesting that the molecules always relax to the same excited state, and that the transition occurs between this excited state and the ground state. In support of this hypothesis, a significant Stokes shift, similar to that found for PCN materials, was observed. Fluorescence quantum yields were then measured for the same excitation wavelengths above $300 \mathrm{~nm}$ (instrumental limit). Results of this analysis were, for 1a: $315 \mathrm{~nm}$ (yield: $0.4 \%$ ); for 2: $304 \mathrm{~nm}(0.5 \%), 353 \mathrm{~nm}(3.4 \%), 367 \mathrm{~nm}$ (2.5\%); for 3: $313 \mathrm{~nm}$ (2.7\%), $356 \mathrm{~nm}$ (14.7\%), $372 \mathrm{~nm} \mathrm{(20.4 \% ).} \mathrm{Whatever} \mathrm{the}$

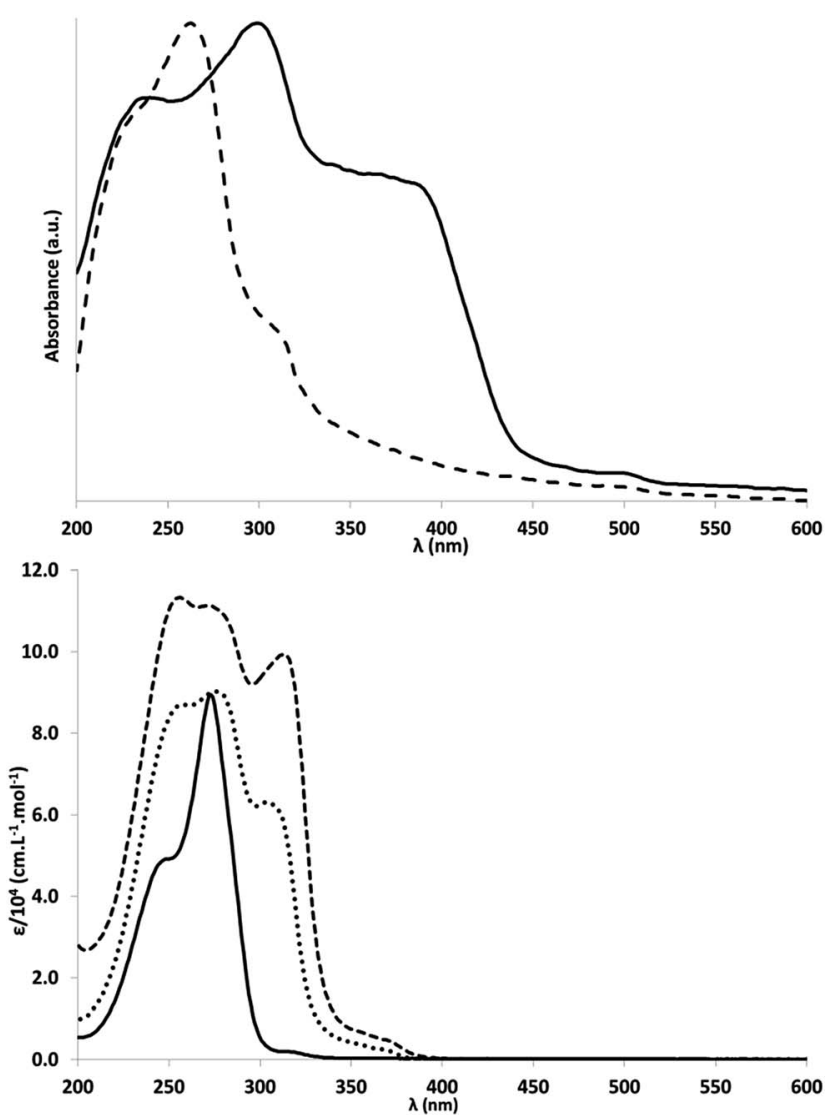

Fig. 2 UV/Vis absorption spectra. Top: Solid state spectra (absorbance) of $\mathrm{g}-\mathrm{CN}$ (solid line) and melem (dashed line). Bottom: 1a (solid line), 2 (dotted line) and 3 (dashed line) in acetonitrile, at room temperature. excitation wavelength, the quantum yield increased for 3 compared to 1a. The values obtained for 3 were similar to those reported for PCN nanosheets (19.6\%) and higher than those found for bulk PCN (4.8\%) under UV illumination. ${ }^{\mathbf{1 0 , 1 2}}$ Both UV/ visible spectra and emission spectra were very similar to those published for colloidal single-layer carbon nitride. ${ }^{3}$

Isobutyl substituents have a rather low effect on the optical properties of 1a compare to melem. For example, the maximum of the emission spectra of melem is given at $366 \mathrm{~nm}$, which is only $16 \mathrm{~nm}$ shifted toward higher energy compare to $1 \mathbf{1 a}^{\mathbf{1 0}} \mathrm{A}$ small shift of $10 \mathrm{~nm}$ is also observed in absorption spectroscopy $\left(\lambda_{\max }=263 \mathrm{~nm}\right.$ for melem and $\lambda_{\max }=273 \mathrm{~nm}$ for $\left.\mathbf{1 a}\right)$.

The absorption wavelengths of the experimental UV-visible spectra were well reproduced by TD-DFT calculations (see ESI: Fig. S33-S42 $\dagger$ ). The main orbitals involved in the lowest energy transition (hereafter referred to as the tail transition) were found to be from external nitrogen and core carbon atoms for monomer 1a (see orbitals in Fig. S46-S57 $\dagger$ ). For compounds 2 and 3 , these orbitals were derived from a combination of external and bridging nitrogen/carbon atoms and core carbon atoms (see ESI for details $\dagger$ ). Further calculations were therefore performed to better assess how both the nature of peripheral groups and their dihedral angle w.r.t. the core influenced the absorption spectra.

UV-Vis spectra calculated for 1a were compared to those calculated for melem (alkyl chain replaced by hydrogen). In these calculations, 1a showed a pronounced bathochromic shift with increasing dihedral angle of the $\mathrm{N}(\mathrm{iBu})_{2}$ groups, modulated by the orbitals involved in the transition: when only heptazine core orbitals were involved, the red-shift was much less pronounced (from $256 \mathrm{~nm}$ at $0^{\circ}$ to $294 \mathrm{~nm}$ at $90^{\circ}$; see Table S5, Fig. S35 and $36 \dagger$ ) than when peripheral groups were involved (tail transition from $322 \mathrm{~nm}$ at a dihedral angle of $0^{\circ}$ to $750 \mathrm{~nm}$ at $90^{\circ}$ ). For melem, the same effect was observed on a smaller scale (from $264 \mathrm{~nm}$ at $0^{\circ}$ to $418 \mathrm{~nm}$ at $75^{\circ}$; the transition probability at $90^{\circ}$ was 0 , see Table S7, Fig. S37 and 38 $\dagger$ ). Thus, the presence of the electron donating iBu alkyl chains leads to a larger red-shift upon increasing the tilt. For dimer 2 (Table S10 and Fig. S40 $\dagger$ ) and dimelem (Table S12 and Fig. S41†), similar effects (offset and rotation-induced red-shifts) were observed for a $30^{\circ}$ rotation of all peripheral ligands, resulting in similar redshifts to those computed for 1a at the same angle.

Because of the almost free rotation around the bridging nitrogen atoms, in our calculations we also varied the relative angle between the two heptazine units (between $0^{\circ}$ and $90^{\circ}$ ), while maintaining the external nitrogen groups coplanar with their respective heptazine cores (see Fig. S40 and 41†). This variation in the relative angle had almost no effect on UV-visible spectra for 2 and dimelem, confirming the relevance of our oligomers: the UV-visible properties of PCN were thus reproduced despite different angles between the heptazine units in the different molecules.

\section{Electrochemistry}

The electrochemical properties of $\mathbf{1 a}, 2$ and $\mathbf{3}$ in acetonitrile were investigated by cyclic voltammetry (Table 1, Fig. 3 and 
Table 1 Electronic properties of oligomers. All potentials are referred to $\mathrm{NHE}^{14}$

\begin{tabular}{|c|c|c|c|c|c|c|c|}
\hline & $\mathrm{E}_{1 / 2}^{\mathrm{rd} a}(\mathrm{~V})$ & $\Delta \mathrm{E}_{\mathrm{p}}^{\mathrm{rd} a}(\mathrm{mV})$ & $\mathrm{E}_{\mathrm{p}}^{\mathrm{oxc}}(\mathrm{V})$ & $\mathrm{E}_{\mathrm{DFT}}^{\mathrm{rd}}(\mathrm{V})$ & $\begin{array}{l}\mathrm{E}_{\mathrm{DFT}}^{\mathrm{OX}} \\
(\mathrm{V})\end{array}$ & $\begin{array}{l}\mathrm{E}_{\mathrm{p}}^{\mathrm{ox}} \\
-\mathrm{E}_{1 / 2}^{\mathrm{rd}}\end{array}$ & $\mathrm{E}_{\mathrm{g}}^{\mathrm{opt} d}(\mathrm{eV})$ \\
\hline 1a & $-2.171^{b}$ & $71^{b}$ & +2.111 & -2.46 & 1.89 & 4.282 & 3.65 \\
\hline 2 & -1.596 & 73 & +2.119 & -1.57 & 1.71 & 3.715 & 3.24 \\
\hline 3 & -1.296 & 61 & +2.113 & -1.32 & 1.70 & 3.409 & 3.16 \\
\hline
\end{tabular}

${ }^{a}$ Quasi-reversible first half-wave reduction potential. ${ }^{b}$ Measured in DMSO. ${ }^{c}$ First irreversible oxidation wave. ${ }^{d} E_{\mathrm{g}}^{\mathrm{opt}}$ values were estimated from the start of the absorption spectra.

S44 $\dagger$ ). Oxidation causes completely irreversible electron transfers in all three compounds, at 2.111, 2.119 and $2.113 \mathrm{~V}$ per NHE, respectively, for the first wave. This first electron transfer was accompanied by decomposition of the corresponding radical cation as, after a first sweep in potential, we observed electrode passivation. Upon reduction, no wave was observed for 1a in acetonitrile as its reduction potential was beyond the electrolyte's electrochemical window (in DMSO, 1a exhibits a quasi-reversible electron transfer at $-2.171 \mathrm{~V}$ per NHE). In acetonitrile, 2 and 3 underwent a first quasi-reversible electron transfer $(-1.632$ and $-1.326 \mathrm{~V}$ per NHE, respectively) followed by a second irreversible reduction reaction, probably associated with a reversible chemical rearrangement. The similar oxidation potentials for 1a, 2 and $\mathbf{3}$ were notable, in contrast the first reduction potential increased significantly with the number of conjugated heptazines. This behaviour, already predicted in linear heptazine models, was rationalized by DFT calculations. ${ }^{13}$ In 1a, 2 and 3, several quasi-degenerate orbitals were found to be close to the HOMO level (Fig. S45†). Peripheral nitrogen

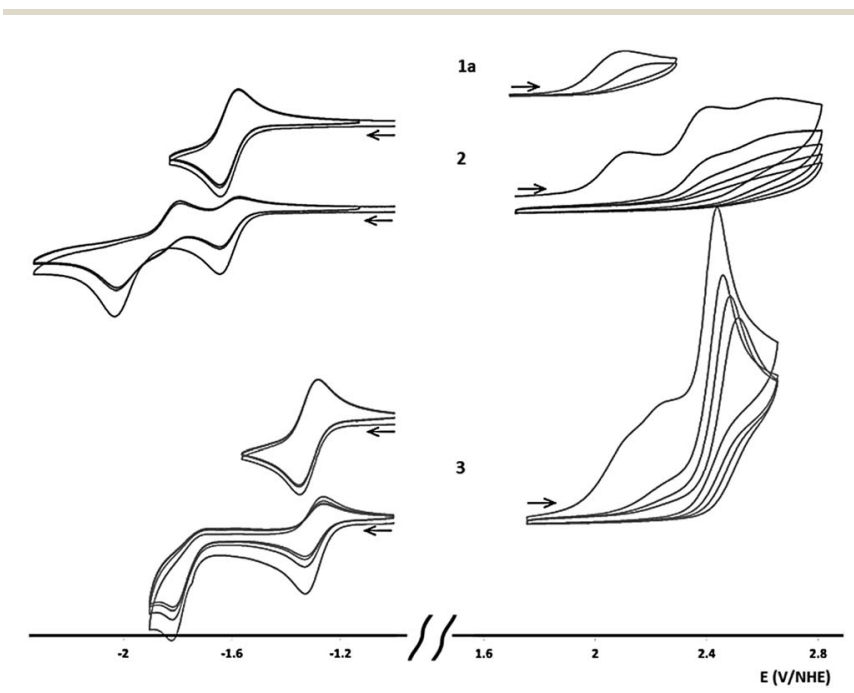

Fig. 3 Cyclovoltamograms for 1a, 2 and 3. Two curves are shown for reduction of 2 and 3, top: scanning only the first reduction wave; bottom: scanning the second reduction wave. Conditions: room temperature, $1 \mathrm{mM}$ compound, vitreous carbon electrode (dia $=3$ $\mathrm{mm})$; electrolyte: $n \mathrm{Bu}_{4} \mathrm{PF}_{6}(0.1 \mathrm{M}) /$ acetonitrile; platinum counterelectrode; scan rate: $100 \mathrm{mV} \mathrm{s}^{-1}$. Arrow indicates initial potential and scanning direction. substituents and low delocalization on the heptazine units significantly contributed to the occupied orbitals closest to the HOMO. Consequently, their energies did not vary significantly between 1a and 3, and the corresponding oxidation potentials were similar. DFT analysis also showed that the LUMO level for the heptazine oligomers was composed of one energetically isolated orbital to which all heptazine cores contributed (through delocalization). The reducing electron would therefore be delocalized over all the heptazine units, facilitating the reduction process when greater numbers of heptazine units are linked. This orbital ladder structure is strongly reminiscent of the band structure reported for g-CN, and is also found in other oligomers composed of several linked delocalized units. ${ }^{2 c}$

\section{Analysis of properties}

The properties of oligomers are often investigated as a function of their size by plotting their physico-chemical characteristics as a function of $1 / N, N$ being the number of core unit(s). ${ }^{16}$ In this representation ( $N \rightarrow$ inf, $1 / N \rightarrow 0$ ), even if some "saturation" effects are omitted, the intercept values resulting from linear interpolations can be reliably used to estimate properties such as band gap, UV-visible transitions, etc. for an equivalent ideal infinite polymer. This method has already been used to evaluate

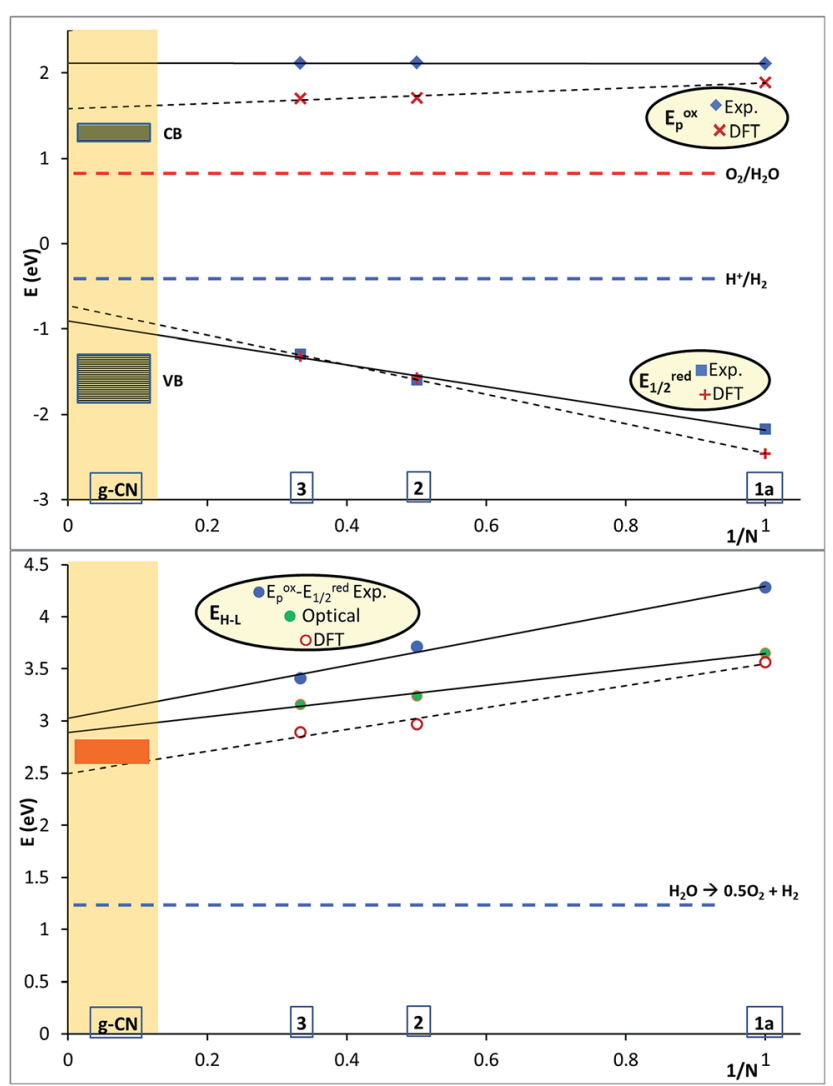

Fig. 4 Energy diagram plotting reduction and oxidation potentials (top) as well as optical gaps (bottom) for 1a, 2, 3 as a function of the inverse of the number of heptazine units $(N)$. CB and VB for $\mathrm{g}-\mathrm{CN}$ were positioned based on ref. $15 . \mathrm{O}_{2}$ and $\mathrm{H}_{2}$ redox potentials were calculated at $\mathrm{pH}=7$. 
band gap of heptazine model by DFT calculation. ${ }^{17}$ We used this method to compare the properties of the heptazine oligomers studied in this work to those of PCN materials (Fig. 4). A caveat of this comparison is that the chemical structure of an infinite polymer based on our oligomers would be expected to be quite different from that of $\mathrm{PCN}$, and the presence of $\mathrm{iBu} / n \mathrm{Bu}$ groups rather than $\mathrm{H}$ on peripheral nitrogen groups could also modify some physical and chemical properties.

The oxidation potential for compounds 1a, 2 and 3 extrapolated to $2.12 \mathrm{~V}$, and the reduction potential extrapolated to $-0.91 \mathrm{~V}$, i.e., a (electrochemical) gap of $3.03 \mathrm{eV}$ for the putative infinite polymer. This extrapolated value was only slightly greater than the optical gap extrapolated based on the UVvisible spectrum $(2.89 \mathrm{eV}, 430 \mathrm{~nm})$. Extrapolated DFT values provided comparable, but slightly smaller values (electrochemical gap: $2.31 \mathrm{eV}$ and optical gap: $2.49 \mathrm{eV}, 498 \mathrm{~nm}$ ). The extrapolated properties for the putative infinite polymer were surprisingly similar to the gap calculated for bulk PCN $(2.6 \mathrm{eV})^{2 c}$ and its optical gap $(2.67 \mathrm{eV}, 464 \mathrm{~nm}) .{ }^{15}$ These results strongly suggest a heptazine structure for PCN rather than a triazine structure. ${ }^{13,18}$ It will be interesting to perform the same kind of physico-chemical studies on the triazine oligomers already described in literature. ${ }^{19}$

\section{Conclusions}

This article reports the synthesis of soluble heptazine oligomers for the first time. These oligomers are surprisingly good molecular models of PCN, and the addition of peripheral groups does not appear to significantly change the properties of the heptazine cores. These perfectly characterised molecules display interesting features, such as strong fluorescence and high absorbance in the UV region with a significant red-shift as the number of linked heptazines increases. Our spectroscopic results are consistent with the oligomers studied being described as "loosely interacting" monomer units, in line with the conclusions of a recent photophysical study of bulk PCN. ${ }^{20}$ Our data and their subsequent extrapolation suggest that sizeable optical absorption in the visible range is unlikely to appear for pure linear heptazine oligomers until a high molecular mass has been reached, in agreement with theoretical studies of linear heptazine structures. ${ }^{\mathbf{1 3}}$ The recently reported visible absorption data for di- and trimelem fractions could therefore be attributed either to additional "impurities" (such as alternative peripheral groups) or to solid effects. ${ }^{7}$ Finally, our oligomers are readily derivatized, opening up new possibilities for heptazine chemistry, making it possible to incorporate g-CNlike properties at will in organic molecules or in coordination complexes.

\section{Acknowledgements}

This work was supported by the French Environment and Energy Management Agency (ADEME) and the CEA's DSM Energy program (MECANICS grant). We thank Dr Maighread Gallagher-Gambarelli for advice on English language usages.

\section{Notes and references}

1 J. Liebig, Ann. Pharm., 1834, 10, 1.

2 (a) Y. Wang, X. Wang and M. Antonietti, Angew. Chem., Int. Ed., 2012, 51, 68; (b) F. Su, M. Antonietti and X. Wang, Catal. Sci. Technol., 2012, 2, 1005; (c) X. Wang, K. Maeda, A. Thomas, K. Takanabe, G. Xin, J. M. Carlsson, K. Domen and M. Antonietti, Nat. Mater., 2009, 8, 76; (d) A. Thomas, A. Fischer, F. Goettmann, M. Antonietti, J.-O. Mueller, R. Schloegl and J. M. Carlsson, J. Mater. Chem., 2008, 18, 4893; (e) F. Goettmann, A. Thomas and M. Antonietti, Angew. Chem., Int. Ed., 2007, 46, 2717.

3 Y. V. Panasiuk, A. E. Raevskaya, O. L. Stroyuk, P. M. Lytvyn and S. Y. Kuchmiy, RSC Adv., 2015, 5, 46843.

4 (a) C. E. Redemanann and D. H. J. Lucas, J. Am. Chem. Soc., 1940, 62, 842; (b) L. Pauling and J. H. Sturdivant, Proc. Natl. Acad. Sci. U. S. A., 1937, 23, 615.

5 B. V. Lotsch, M. Doeblinger, J. Sehnert, L. Seyfarth, J. Senker, O. Oeckler and W. Schnick, Chem.-Eur. J., 2007, 13, 4969.

6 L. Chen, Y. Hernandez, X. Feng and K. Muellen, Angew. Chem., Int. Ed., 2012, 51, 7640.

7 V. W. H. Lau, M. B. Mesch, V. Duppel, V. Blum, J. Senker and B. V. Lotsch, J. Am. Chem. Soc., 2015, 137, 1064.

8 (a) H. Schroeder and E. Kober, J. Org. Chem., 1962, 27, 4262; (b) J. Li, H. Nomura, H. Miyazaki and C. Adachi, Chem. Commun., 2014, 50, 6174; (c) E. Wirnhier and W. Schnick, Eur. J. Inorg. Chem., 2012, 1840; (d) A. Schwarzer, U. Boehme and E. Kroke, Chem.-Eur. J., 2012, 18, 12052; (e) T. Saplinova, C. Lehnert, U. Boehme, J. Wagler and E. Kroke, New J. Chem., 2010, 34, 1893; (f) T. Saplinova, V. Bakumov, T. Gmeiner, J. Wagler, M. Schwarz and E. Kroke, Z. Anorg. Allg. Chem., 2009, 635, 2480; $(g)$ B. Traber, T. Oeser, R. Gleiter, M. Goebel and R. Wortmann, Eur. J. Org. Chem., 2004, 4387; (h) D. R. Miller, D. C. Swenson and E. G. Gillan, J. Am. Chem. Soc., 2004, 126, 5372; ( $i$ ) E. Kroke, M. Schwarz, E. HorathBordon, P. Kroll, B. Noll and A. D. Norman, New J. Chem., 2002, 26, 508; (j) A. Schwarzer, T. Saplinova and E. Kroke, Coord. Chem. Rev., 2013, 257, 2032.

9 A. Schwarzer and E. Kroke, New J. Chem., 2011, 35, 953.

10 B. Jurgens, E. Irran, J. Senker, P. Kroll, H. Muller and W. Schnick, J. Am. Chem. Soc., 2003, 125, 10288.

11 (a) M. Oki, Applications of Dynamic NMR Spectroscopy to Organic Chemistry, Deerfield Beach, Florida, USA, 1985; (b) J. Sandström, Dynamic NMR spectroscopy, London, 1982.

12 X. Zhang, X. Xie, H. Wang, J. Zhang, B. Pan and Y. Xie, J. Am. Chem. Soc., 2013, 135, 18.

13 C. Butchosa, P. Guiglion and M. A. Zwijnenburg, J. Phys. Chem. C, 2014, 118, 24833.

14 V. V. Pavlishchuk and A. W. Addison, Inorg. Chim. Acta, 2000, 298, 97.

15 J. S. Zhang, X. F. Chen, K. Takanabe, K. Maeda, K. Domen, J. D. Epping, X. Z. Fu, M. Antonietti and X. C. Wang, Angew. Chem., Int. Ed., 2010, 49, 441.

16 (a) J. Gierschner, J. Cornil and H.-J. Egelhaaf, Adv. Mater., 2007, 19, 173; (b) J. Rissler, Chem. Phys. Lett., 2004, 395, 92; 
(c) H. Meier, U. Stalmach and H. Kolshorn, Acta Polym., 1997, 48, 379.

17 P. Chamorro-Posada, J. Vazquez-Cabo, F. M. SanchezArevalo, P. Martin-Ramos, J. Martin-Gil, L. M. Navas-Gracia and R. C. Dante, J. Solid State Chem., 2014, 219, 232.

18 G. Algara-Siller, N. Severin, S. Y. Chong, T. Bjorkman, R. G. Palgrave, A. Laybourn, M. Antonietti, Y. Z. Khimyak, A. V. Krasheninnikov, J. P. Rabe, U. Kaiser, A. I. Cooper, A. Thomas and M. J. Bojdys, Angew. Chem., Int. Ed., 2014, 53, 7450 .
19 W. J. Schnabel, R. Ratz and E. Kober, J. Org. Chem., 1962, 27, 2514.

20 (a) C. Merschjann, T. Tyborski, S. Orthmann, F. Yang, K. Schwarzburg, M. Lublow, M. C. Lux-Steiner and T. Schedel-Niedrig, Phys. Rev. B: Condens. Matter Mater. Phys., 2013, 87, 205204; (b) T. Tyborski, C. Merschjann, S. Orthmann, F. Yang, M. C. Lux-Steiner and T. SchedelNiedrig, J. Phys.: Condens. Matter, 2012, 24, 162201. 